\section{OP0191 \\ EPIGENETIC PROFILING OF SYNOVIAL FIBROBLASTS REVEALS STRUCTURAL DNA DYNAMICS AT DISEASE IMPLICATED CHROMOSOME REGIONS}

Xiangyu $\mathrm{Ge}^{1}$, Mojca Frank-Bertoncelj ${ }^{2}$, Amanda Mcgovern ${ }^{1}$, Gisela Orozco ${ }^{1}$, Paul Martin ${ }^{1}$, Caroline Ospelt ${ }^{2}$, Stephen Eyre'. 'Versus Arthritis Centre for Genetics and Genomics, School of Biological Sciences, Faculty of Biology, Medicine and Health, The University of Manchester, UK, Manchester, United Kingdom; ${ }^{2}$ University Hospital Zürich, Zurich, Switzerland

Background: One of the more striking findings from genome-wide association studies (GWAS) is how the majority of disease associated genetic variants are found within gene regulatory regions, known as enhancers. It is now well established that these enhancers regulate target genes through physical interactions. We, and others, have shown that these interactions can vary between cell types and act over long distances. Therefore, if we are to fully translate GWAS findings, we need link disease associated enhancers to their target genes, in the relevant cell types. So far we only have a limited picture in rheumatoid arthritis (RA) relevant cell types, since synovial fibroblasts (SF) have been omitted from this type of chromatin interaction analysis.

Objectives: Investigate dynamic $\mathrm{Hi}-\mathrm{C}$ interactions in unstimulated and stimulated SF, mapping RA enhancers to their target genes, and correlating with gene expression.

Methods: We cultured SF from synovial tissues of RA patients. After stimulation of the SF $(n=6)$ with $10 \mathrm{ng} / \mathrm{ul}$ TNF for $24 \mathrm{~h}, \mathrm{Hi}-\mathrm{C}$ and RNA libraries were generated and sequenced on the Illumina HiSeq 4000 . We called dynamic active and inactive regions of the genome ( $A / B$ compartments), mapped on RA associated enhancers, linked these to target genes and correlated the interactions with dynamic expression.

Results: We found a region on the chromosome $6 \mathrm{q} 23$, intergenic between OLIG3 and TNFAIP3, with an enhancer containing SNPS associated with RA to be dynamically linked to the TNFAIP3 gene through DNA activity, interactions and corresponding gene expression.

As shown in figure 1a, the genomic region containing the RA associated variants (red square) only resides in an open, active region of DNA (black bar, compartment A) upon TNF-stimulation of the SF. This region then makes a strong interaction with the promoter of TNFAIP3 (figure 1a) in stimulated cells, which corresponds to a more than 100-fold increase in TNFAIP3 gene expression (figure 1b).

Conclusion: Using independent data, we have indicated how the RA region on $6 q 23$ could influence the expression of TNFAIP3, under stimulatory conditions in SF cells. This supports previous work, where this particular $6 \mathrm{q} 23$ enhancer is preferentially active in RA synovium, compared to osteoarthritis, reflecting the different stimulatory conditions in each disease (Ai et al. 2018). We have successfully linked an RA associated enhancer to its target gene in SF, indicating how the enhancer works over a large distance to interact with and regulate the TNFAIP3 gene. Furthermore we show that this enhancer containing RA associated SNPs is only active upon stimulation with TNF in SF.

REFERENCE:

[1] Ai R, et al. Nat Commun. 2018 May 15;9(1):1921

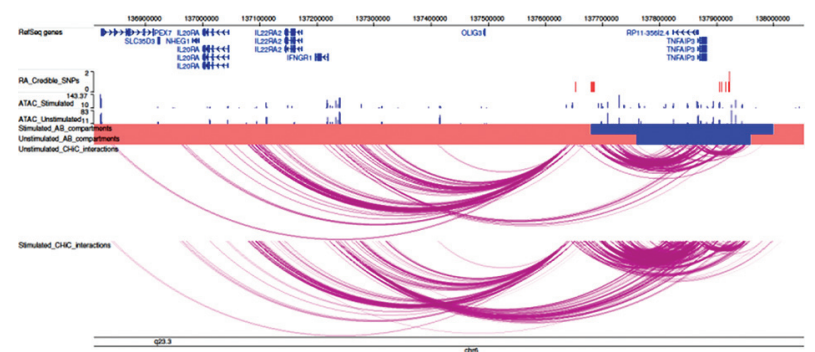

Figure 1a

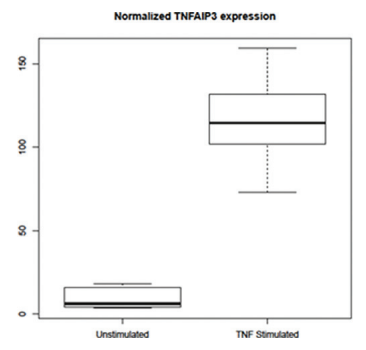

Figure 1b
Disclosure of Interests: None declaredDOI: 10.1136/annrheumdis-2019eular.4094

\begin{tabular}{|l|l}
\hline OP0192 & DISSECTING THE LONG-RANGE GENE REGULATION \\
OF RHEUMATOID ARTHRITISRISK ENHANCERS AT \\
THE 5Q11 LOCUS USING THE COMPLEMENTARY \\
APPROACHES OF CRISPRA AND CRISPRI
\end{tabular}

Kate Duffus ${ }^{1}$, Oliver Gough ${ }^{1}$, Gisela Orozco ${ }^{1}$, Anthony Adamson ${ }^{2}$, Stephen Eyre ${ }^{1}$. ${ }^{1}$ Arthritis Research UK Centre for Genetics and Genomics, School of Biological Sciences, Faculty of Biology, Medicine and Health, The University of Manchester, Manchester, United Kingdom; ${ }^{2}$ Faculty of Biology, Medicine and Health University of Manchester, Manchester, United Kingdom

Background: Large-scale genome wide association studies (GWAS) in rheumatoid arthritis (RA) have revealed that the majority of associated risk variants lie in non-coding regions of the genome. For $\mathrm{RA}$ it has been demonstrated that risk variants are significantly enriched in T cell specific enhancers. One such susceptibility locus known as $5 q 11$ is the third strongest association with RA. At this locus the lead risk variants lie in two distinct active enhancer elements intronic to the ANKRD55 gene. Previous work using chromatin conformation has shown these enhancers make physical contact with the promoter of IL6ST, $150 \mathrm{~kb}$ away on the linear chromosome ${ }^{1}$. Whilst $A N K R D 55$ is a gene of unknown function, IL6ST is part of the IL6R complex which regulates the IL6 signalling pathway. Tocilizumab is already an approved drug for RA, which works against the IL6R. CRISPR is an ideal tool to dissect this enhancer-gene relationship to provide us with empirical evidence that the intronic enhancer within $A N K R D 55$ regulates the expression of IL6ST. Understanding how RA risk genetic background affects gene regulation will give us a better understanding of the underlying biology of the disease and can ultimately be used to help repurpose or discover novel drug therapies.

Objectives: To use CRISPR interference (CRISPRi) and CRISPR activation (CRISPRa) to perturb the ANKRD55 intronic enhancer and measure the downstream effect on the expression of IL31RA, IL6ST, ANKRD55).

Methods: We used dead (dCas9) a modified CRISPR Cas9 enzyme that is catalytically inactive, that precisely targets DNA, but does not cut. Instead, effecto molecules are fused to the dCas9 (VP64 for activation and KRAB for repression) to alter the transcription of the targeted genes. Two lead functional risk SNPs at the $5 q 11$ risk locus $r s 10065637$ and $r s 7731626\left(R^{2}=0.4472\right)$ lie in two distinct enhancer elements separated by $5.5 \mathrm{~kb}$. We designed 4 guides across each enhancer, and transduced a $\mathrm{T}$ cell line (Jurkat) using lentiviral delivery; first dCas9-KRAB and dCas9-VP64 and subsequently the pool of guides for each enhancer alongside controls. We cultured the cells until confluent and then sorted the top $60 \%$ expression of BFP-GFP (KRAB) or MCherry-GFP (VP64). RNA was extracted and then a qPCR was performed (Quantstudio) for IL31RA, IL6ST and ANKRD55.

Results: In the CRISPRa Jurkat cells the guides targeted to both SNP containing enhancers significantly increased the expression of ANKRD55: rs7731626 $(p=<0.0001)$ and $r 6659219(p=0.0003)$ respectively. They also increased the expression of IL6ST rs7731626 (p 0.0023) and rs6859219 ( $\mathrm{p}=0.01)$. In CRISPR Jurkat cells the guides targeted to both SNP containing enhancers significantly decreased the expression of ANKRD55: rs7731626 ( $\mathrm{p}=<0.001)$ and rs6859219 $\mathrm{p}=(0.0071)$. When guides were simultaneously used for both enhancers rs6859219+rs7731626 there was also a significant decrease in expression of ANKRD55. $(\mathrm{p}=<0.0001)$. Only the pool guides against both enhancers significantly decreased the expression of $I L 6 S T(p=<0.0001)$. In both systems no significant effect was seen on the expression of IL31RA (Figure 1).

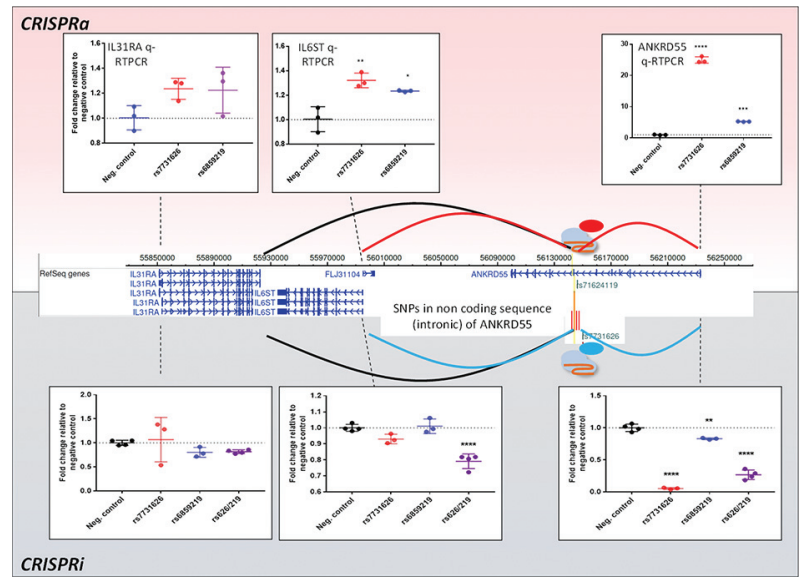

Figure 1 
Conclusion: Results from CRISPRi and CRISPRa are concordant and support the idea that intronic enhancers within ANKRD55, containing SNPs associated with RA susceptibility, can regulate the expression of both ANKRD55 and also modulate long range gene regulation with IL6ST to influence disease risk. These techniques are crucial to begin to dissect and translate GWAS findings. REFERENCE:

[1] Capture Hi-C reveals novel candidate genes and complex long-range interactions.

Disclosure of Interests: None declared

DOI: 10.1136/annrheumdis-2019-eular.7946

\section{OP0193 CAMP RESPONSE ELEMENT MODULATOR (CREM)A INDUCES DUAL SPECIFICITY PROTEIN PHOSPHATASE (DUSP)4 THROUGH EPIGENETIC REMODELING, PROMOTING IL-17A AND REDUCING IL-2 EXPRESSION IN T CELLS}

Sigrun Hofmann ${ }^{1}$, Katrin Maebert ${ }^{1}$, Franz Kapplusch ${ }^{2}$, Susanne Russ ${ }^{1}$, Christian Hedrich ${ }^{1,2,3}$. 'Universitaetsklinikum Carl Gustav Carus, TU Dresden, Paediatrics, Dresden, Germany, ${ }^{2}$ Institute of Translational Medicine, University of Liverpool, Department of Women's and Children's Health, Liverpool, United Kingdom; ${ }^{3}$ Alder Hey Children's NHS Foundation Trust Hospital, Department of Paediatric Rheumatology, Liverpool, United Kingdom

Background: Tissue inflammation and organ damage in systemic lupus erythematosus (SLE) have been linked to effector $T$ cells that are characterized by increased IL-17A and reduced IL-2 production(1). T cells from patients with SLE express increased levels of the transcription factor cAMP response element modulator (CREM) $\alpha$ that contributes to altered cytokine expression(1-3). However the exact molecular events contributing to dysregulated IL-17A and IL-2 expression are incompletely understood.

Objectives: To investigate molecular events that promote effector $\mathrm{T}$ cells in health and disease. The definition of molecular regulators of effector $\mathrm{T}$ cell generation and activity may deliver new biomarkers and potential therapeutic targets in disorders characterized by altered effector T cell function, including (but not limited to) SLE.

Methods: Using CRISPR/Cas9 genome editing and lentiviral transduction, we generated CREM $\alpha$ deficient or overexpressing Jurkat T cells. Gene expression profiles in Jurkat and primary human CD4+ T cells were assessed by qRT-PCR and $\mathrm{mRNA}$ probe-based hybridization techniques. Gene regulation events were investigated using luciferase reporter assays (trans-activation) and ChIP. Interactions between CREM $\alpha$ and the transcriptional co-activator p300 were assessed using proximity ligation assays and p300 knock-down with siRNAs.

Results: We link CREM $\alpha$ production in effector $C D 4^{+} T$ cells with increased expression of dual specificity protein phosphatase (DUSP)4. Using genetically modified Jurkat T cells, we demonstrate that CREM $\alpha$ induces DUSP4 through recruitment of the transcriptional co-activator p300 and histone H3K18 acetylation. Using DUSP4 transfection models and genetically modified Jurkat $T$ cells, we support previous reports suggesting that DUSP4 induces IL-17A while limiting IL-2 expression. Furthermore, we demonstrate that $\mathrm{CD} 4^{+} \mathrm{T}$ cells from patients with juvenile-onset SLE share the phenotype with CREM $\alpha$ over-expressing $\mathrm{CD}^{+} \mathrm{T}$ cells with increased DUSP4 expression that contributes to imbalanced IL-17A and IL-2 production.

Conclusion: Collectively, our results deliver previously unknown CREM $\alpha$-mediated molecular mechanisms promoting effector $T$ cells and support the central involvement of CREM $\alpha$ in the pathophysiology of SLE. CREM $\alpha$ and DUSP 4 may prove valuable as disease biomarkers and/or targets in the search for individualized and target-directed treatments.

\section{REFERENCES:}

[1] SLE-Associated Defects Promote Altered T Cell Function. Crispin JC, Hedrich CM, et al. Crit Rev Immunol. 2017;37(1):39-58.

[2] cAMP responsive element modulator: a critical regulator of cytokine production. Rauen T, Hedrich CM, et al. Trends Mol Med. 2013 Apr;19 (4):262-9.

[3] cAMP response element modulator $\alpha$ controls IL2 and IL17A expression during CD4 lineage commitment and subset distribution in lupus. Hedrich CM, Crispin JC, et al. Proc Natl Acad Sci U S A. 2012 Oct 9;109 (41):16606-11.

Acknowledgement: This is a summary of independent research funded by the Fritz-Thyssen-Foundation and carried out at the National Institute of Health Research (NIHR) Alder Hey Clinical Research Facility. The views expressed are those of the authors.

Disclosure of Interests: Sigrun Hofmann: None declared, Katrin Maebert: None declared, Franz Kapplusch: None declared, Susanne Russ: None declared, Christian Hedrich Grant/research support from: Novartis Pharmaceuticas for
Research study on effector T cells in psoriasis, Speakers bureau: In 2016: Roche Pharmaceuticals, RheumatoLogisch, Dresden, Germany; Novartis, Advisory board > Travel costs.

DOI: 10.1136/annrheumdis-2019-eular.999

\section{OP0194 \\ HISTONE DEACETYLASE 1 (HDAC1): A KEY MEDIATOR OF T CELLS FOR THE PATHOGENESIS OF RHEUMATOID ARTHRITIS}

Lisa Göschl ${ }^{1}$, Victoria Saferding ${ }^{1}$, Nicole Boucheron ${ }^{2}$, Johan Backlund ${ }^{3}$, Alexander Platzer ${ }^{1}$, Kiyoshi Hirahara ${ }^{4}$, Han-Yu Shih ${ }^{5}$, Patrick Matthias ${ }^{6}$, Clemens Scheinecker ${ }^{1}$, Günter Steiner ${ }^{1}$, Wilfried Ellmeier ${ }^{2}$, Michael Bonelli ${ }^{1}$. ${ }^{1}$ Division of Rheumatology, Department of Internal Medicine III, Medical University of Vienna, Vienna, Austria; ${ }^{2}$ Institute of Immunology, Division of Immunobiology, Vienna, Austria; ${ }^{3}$ Department of Medical Biochemistry and Biophysics, Medical Inflammation Research, Karolinska Institute, Stockholm, Sweden; ${ }^{4}$ Graduate School of Medicine, Chiba University, Department of Immunology, Chiba, Japan; ${ }^{5}$ National Institutes of Health, National Institute of Arthritis, Bethesda, United States of America; ${ }^{6}$ Friedrich Miescher Institute for Biomedical Research, Basel, Switzerland

Background: Despite enormous efforts to develop new therapeutic strategies for treatment of rheumatoid arthritis (RA), the large number of non responding patients to currently available drugs underlies the unmet need to identify new therapeutic targets. Certain $\mathrm{CD} 4^{+} \mathrm{T}$ cell subsets, especially those polarized toward the T helper (Th) subsets Th1 andTh17, have been shown to be major drivers of inflammation in patients with RA. The expression of their key transcription factors is controlled by histone modifications which includes acetylation of lysine residues mediated by histone deacetylases (HDAC). Indeed, pan HDAC inhibitors have been shown to be a potential therapeutic strategy. However, major side effects limited the clinical use and underline the need of more specific HDAC inhibitors. Objectives: We addressed the individual role of HDAC1 on the development of collagen-induced arthritis model (CIA), which partially reflects human $\mathrm{RA}$.

Methods: Mice with a T cell specific deletion of HDAC1 (HDAC1 cKO) were generated by using the CD4Cre/LoxP system. At week 8 of age arthritis was induced in wild type (WT) and HDAC1 cKO mice by immunizing with chicken collagen II (CII), emulsified in complete Freund's adjuvant. After 21 days mice received a booster injection of CII. The animals were 3 times per week scored for paw swelling and grip strength. Anti-CII antibody levels were determined by ELISA. Various cell subsets, including Th cells, where detected in the blood, the spleen and the draining lymph node by FACS analysis. To test antigen-specific T cell activation we performed in vitro restimulation of spleen and lymph node cells with collagen II followed by assessment of cytokine production and quantification of the proliferation rate using $3 \mathrm{H}$ thymidine incorporation.

After 4 and after 10 weeks mice were sacrificed and paraffin sections of the affected joints were analyzed for histomorphologic signs of inflammation, cartilage and bone destruction.

Results: Eighty percent of the animals developed serum anti-Cll anibodies (IgM and $\lg G$ ) whereby the antibody levels were a day 21 of disease similar between the HDAC1 $\mathrm{cKO}$ and the WT mice. Furthermore, no differences in the production of antibody subclasses, especially of pathogenic IgG2c antibodies, were observed. Enhanced percentages of Th1 and Th17 cells among HDAC1-null $\mathrm{CD} 4^{+} \mathrm{T}$ cells were detected after immunization in the HDAC1 cKO mice. Nonetheless and unexpectedly, these mice did not develop any signs of disease at the clinical level while WT mice developed pronounced paw swelling and loss of grip strength. In accordance with the clinical data, histological analysis revealed no signs of inflammation, no bone erosion and no appearance of osteoclasts in the joints of HDAC1 cKO mice. This appeared to be mainly caused by an impaired migratory capacity of HDAC1 $\mathrm{CKO} \mathrm{CD}^{+} \mathrm{T}$ cells, so that they were unable to invade the joints.

Conclusion: Our data show the importance of HDAC1 as a key immune regulator in the pathogenesis of $\mathrm{T}$ cell driven collagen induced arthritis. Therefore it might be considered as an interesting novel therapeutic target in RA.

Disclosure of Interests: None declared

DOI: 10.1136/annrheumdis-2019-eular.5619

OP0195

\section{SELECTIVE EXPANSION OF REGULATORY T-CELLS IN HUMANS BY A NOVEL IL-2 CONJUGATE T-REG STIMULATOR, NKTR-358, BEING DEVELOPED FOR THE TREATMENT OF AUTOIMMUNE DISEASES}

Christie Fanton ${ }^{1}$, Suresh Siddhanti ${ }^{1}$, Neha Dixit ${ }^{1}$, Lin Lu $^{1}$, Toufigh Gordi ${ }^{1}$, Daniel Dickerson ${ }^{2}$, Jonathan Zalevsky ${ }^{1}$, Brian Kotzin ${ }^{1} .{ }^{1}$ Nektar Therapeutics, San Francisco, United States of America; ${ }^{2}$ PRA Health Sciences, Lenexa, United States of America

Background: Impaired IL-2 production and dysfunction of regulatory $\mathrm{T}$ cells (Tregs) have been identified as key immunological defects leading to the 